\title{
Binding of an oligomeric ellagitannin series to bovine serum albumin (BSA): analysis by isothermal titration calorimetry (ITC)
}

Article

Accepted Version

Karonen, M., Oraviita, M., Mueller-Harvey, I., Salminen, J.-P. and Green, R. J. (2015) Binding of an oligomeric ellagitannin series to bovine serum albumin (BSA): analysis by isothermal titration calorimetry (ITC). Journal of Agricultural and Food Chemistry, 63 (49). pp. 10647-10654. ISSN 0021-8561 doi: https://doi.org/10.1021/acs.jafc.5b04843 Available at https://centaur.reading.ac.uk/47797/

It is advisable to refer to the publisher's version if you intend to cite from the work. See Guidance on citing.

To link to this article DOI: http://dx.doi.org/10.1021/acs.jafc.5b04843

Publisher: American Chemical Society

All outputs in CentAUR are protected by Intellectual Property Rights law, including copyright law. Copyright and IPR is retained by the creators or other copyright holders. Terms and conditions for use of this material are defined in the End User Agreement. 


\section{www.reading.ac.uk/centaur}

\section{CentAUR}

Central Archive at the University of Reading

Reading's research outputs online 


\section{Article}

Subscriber access provided by READING UNIV

\section{Binding of an Oligomeric Ellagitannin Series to BSA: Analysis by Isothermal Titration Calorimetry}

Maarit Karonen, Marianne Oraviita, Irene Mueller-Harvey, Juha-Pekka Salminen, and Rebecca Joanne Green J. Agric. Food Chem., Just Accepted Manuscript • DOI: 10.1021/acs.jafc.5b04843 • Publication Date (Web): 26 Nov 2015

Downloaded from http://pubs.acs.org on November 30, 2015

\section{Just Accepted}

"Just Accepted" manuscripts have been peer-reviewed and accepted for publication. They are posted online prior to technical editing, formatting for publication and author proofing. The American Chemical Society provides "Just Accepted" as a free service to the research community to expedite the dissemination of scientific material as soon as possible after acceptance. "Just Accepted" manuscripts appear in full in PDF format accompanied by an HTML abstract. "Just Accepted" manuscripts have been fully peer reviewed, but should not be considered the official version of record. They are accessible to all readers and citable by the Digital Object Identifier (DOI®). "Just Accepted" is an optional service offered to authors. Therefore, the "Just Accepted" Web site may not include all articles that will be published in the journal. After a manuscript is technically edited and formatted, it will be removed from the "Just Accepted" Web site and published as an ASAP article. Note that technical editing may introduce minor changes to the manuscript text and/or graphics which could affect content, and all legal disclaimers and ethical guidelines that apply to the journal pertain. ACS cannot be held responsible for errors or consequences arising from the use of information contained in these "Just Accepted" manuscripts. 


\section{Binding of an Oligomeric Ellagitannin Series to BSA: Analysis by Isothermal Titration Calorimetry}

Maarit Karonen $^{*, \dagger}$, Marianne Oraviita ${ }^{\dagger}$, Irene Mueller-Harvey ${ }^{\ddagger}$, Juha-Pekka Salminen ${ }^{\dagger}$, and Rebecca J. Green ${ }^{*} \S$

${ }^{\dagger}$ Department of Chemistry, University of Turku, Vatselankatu 2, Turun Yliopisto, Turku FI-20014, Finland

${ }^{\ddagger}$ School of Agriculture, Policy and Development, University of Reading, Earley Gate, P.O. Box 236, Reading RG6 6AT, United Kingdom

${ }^{\S}$ School of Chemistry, Food and Pharmacy, University of Reading, Whiteknights, P.O. Box 224, Reading RG6 6AP, United Kingdom

* Corresponding authors, (M.K.) Tel: + 358-2-333-6830; Fax: +358-2-333-6700; E-mail: maarit.karonen@utu.fi. (R.J.G) Tel: +44-118-378-8446; Fax: +44-118-378-6562; E-mail: rebecca.green@reading.ac.uk. 
1 ABSTRACT:

2 A unique series of oligomeric ellagitannins was used to study their interactions with bovine serum

3 albumin (BSA) by isothermal titration calorimetry. Oligomeric ellagitannins, ranging from

4 monomer up to heptamer and a mixture of octamer-undecamers, were isolated as individual pure

5 compounds. This series allowed studying the effects of oligomer size and other structural features.

6 The monomeric to trimeric ellagitannins deviated most from the overall trends. The interactions of

7 ellagitannin oligomers from tetramers to octa-undecamers with BSA revealed strong similarities. In

8 contrast to the equilibrium binding constant, enthalpy showed an increasing trend from the dimer to

9 larger oligomers. It is likely that first the macrocyclic part of the ellagitannin binds to the defined

10 binding sites on the protein surface and then the "flexible tail" of the ellagitannin coats the protein

11 surface. The results highlight the importance of molecular flexibility to maximize binding between

12 the ellagitannin and protein surfaces.

13

14 KEYWORDS: Interactions, molecular size, polyphenol, protein, thermodynamics

15

16

17 


\section{INTRODUCTION}

Plants produce a variety of secondary metabolites including polyphenols in their tissues to protect them for example against pathogens and insect herbivores. The most complicated polyphenol structures are tannins which by definition have the ability to bind and precipitate proteins. Tannins can be divided into condensed tannins, hydrolysable tannins (gallotannins and ellagitannins) in addition to phlorotannins, which are found only in algae. For decades, ellagitannins were an underestimated class of bioactive plant tannins. ${ }^{l}$ However, ellagitannins are one of the most promising tannin classes with potent biological activities, including antimicrobial and antioxidant activities. ${ }^{1,2}$ More than 1000 individual ellagitannins have been identified from plants and natural ellagitannins larger than pentamers were recently reported. ${ }^{3-5}$

Dietary tannins can affect animal nutrition and health in several ways, for example through enabling a better utilization of feed proteins, generating anthelmintic effects against gastrointestinal nematodes and by lowering nitrogenous and methane emissions. ${ }^{6-16}$ Tannins may bind dietary proteins and thus reduce the degradation of these proteins in the rumen and may also enhance the amount of protein available for digestion in the small intestine. Tannins can form soluble and/or insoluble complexes with proteins and the tannin-protein interactions are both

35 tannin- and protein specific. ${ }^{17}$ Bovine serum albumin (BSA) is a well-characterized model protein and it has been widely used for the investigations of tannin-protein interactions. ${ }^{18}$ Previous results have shown that tannins have higher affinities to loosely structured globular proteins, such as BSA, than to compact globular structures. ${ }^{19}$ thermodynamics of tannin-protein interactions. ITC has both a reference and a sample cell at a constant temperature and the technique relies only on the detection of a heat effect upon binding; it 
43 interactions. $^{20,21}$ In a single ITC experiment, the strength and stoichiometry of the interaction

44 between tannin and protein can be measured, i.e. the enthalpy $\left(\Delta \mathrm{H}_{\mathrm{obs}}\right)$ and stoichiometry of binding

45 (n) and the binding constant $(\mathrm{K})$ can be determined. The binding of condensed tannins and

46 hydrolyzable tannins to proteins has been previously studied by ITC. ${ }^{22-26}$ The binding parameters

47 have been related to the structural flexibility of tannins. ${ }^{24,25}$ Most of the studies have been

48 conducted using condensed tannins. However, the use of condensed tannins as model compounds

49 poses particular problems as the determination of their molecular weight is not straight forward. ${ }^{23}$

50 Oligomeric and polymeric condensed tannins cannot be chromatographically separated and

51 therefore, they are usually isolated and purified as mixtures. ${ }^{27-29}$ Therefore, their molecular weight

52 is an estimation based on the size distribution within a condensed tannin mixture, obtained for

53 example by acidic degradation in the presence of nucleophiles or by mass spectrometry. ${ }^{30-35}$ It is

54 feasible, however, to isolate ellagitannins as pure compounds ${ }^{4,36}$, and this offers a unique 55 opportunity to investigate the effects of different structural features, such as molecular size and 56 monomeric units.

In this study, we utilized ITC to study the interaction between ellagitannins and BSA.

We characterized the thermodynamics of the binding of tellimagrandin I-based oligomeric ellagitannins $^{3,4}$ (Fig. 1) to bovine serum albumin BSA. The ellagitannin oligomers were a unique

60

61

62

63

64

65

66

67 series consisting of tellimagrandin I (monomer), oenothein B (dimer), oenothein A (trimer) plus a tetramer, pentamer, hexamer and heptamer (Fig. 1) and a mixture of octamers to undecamers. These ellagitannin oligomers are excellent model compounds for the ITC studies as they can be isolated as individual pure compounds and are well-characterized with exact molecular weights. ${ }^{3}$ This work is the very first systematic investigation of ellagitannins using an oligomeric series so that we can evaluate the effect of the molecular size and decouple this from other structural features, such as functional groups, which has been a problem when interactions between condensed tannins and proteins were studied. The oligomers consisted of similar monomeric units, which also enabled the 
68

69

70

71

72

73

74

75

76

77

78

79

80

81

82

83

84

85

86

87

88

89

90

91

direct comparison of the interaction between the different oligomers based on the number of monomeric tellimagrandin I units.

\section{MATERIALS AND METHODS}

Materials. Acetone (technical grade) used in the collection of plant materials was from VWR International (Leuven, Belgium). Acetone and methanol (analytical grade) used in the Sephadex LH-20 fractionations, methanol and acetonitrile (HPLC grade) used in the preparative and semipreparative HPLC were obtained from VWR International (Fontenay-Sous-Bois, France). LiChroSolv ${ }^{\circledR}$ acetonitrile (hypergade for LC-MS) was purchased from Merck KGaA (Darmstadt, Germany) and formic acid (eluent additive for LC-MS) was from VWR International Ltd. (Poole, England). The water was filtered through an Elgastat UHQ-PS purification system (Elga, Kaarst, Germany) or with Synergy ${ }^{\circledR}$ UV water purification system (Millipore SAS, Molsheim, France). BSA (purified by heat shock fractionation, $\mathrm{pH} 7$, purity $\geq 98 \%$, lyophilized powder, $66 \mathrm{kDa}$ ) was purchased from Sigma-Aldrich (St. Louis, US).

Isolation and Characterization of Ellagitannins. A series of oligomeric ellagitannins was purified: monomer, dimer, trimer, tetramer, pentamer, hexamer, heptamer and a mixture of chromatographically non-separated octamers to undecamers. ${ }^{3,4}$ The monomeric ellagitannin, tellimagrandin I, was isolated from meadowsweet (Filipendula ulmaria) inflorescences and the other oligomeric ellagitannins from fireweed (Epilobium angustifolium) inflorescences. The inflorescences were collected during summer 2011 from southwest Finland. The plant material was collected and placed directly into ten bottles of $1 \mathrm{~L}$, which were then immediately filled with acetone, transferred to the laboratory, and stored in a cold room $\left(+4{ }^{\circ} \mathrm{C}\right)$ prior the isolation of ellagitannins. 
The extraction and isolation of ellagitannins followed mainly the previously outlined methods. ${ }^{3,4}$ However, some modifications were made in order to enhance and speed up the largescale extractions and fractionations. The acetone extracts of fireweed inflorescences were combined and concentrated to $300-500 \mathrm{~mL}$ of water phase. The concentrated water phases were fractionated twice with Sephadex LH-20 chromatography. First, a rough fractionation was performed in a beaker, and then careful fractionation was performed for a selected fraction by Sephadex LH-20 column chromatography as previously described. ${ }^{37}$ The isolation of ellagitannins from the Sephadex fractions was performed by preparative HPLC. The HPLC-DAD system consisted of a Waters Delta 600 liquid chromatograph, a Waters 600 Controller, a Waters 2998 Photodiode Array Detector and a Waters Fraction Collector III. The column (approximately $327 \mathrm{~mm} \times 33 \mathrm{~mm}$ ) was manually filled with LiChroprep RP-18 (40-63 $\mu \mathrm{m})$ material (Merck KGaA, Darmstadt, Germany). The flow rate was $8 \mathrm{~mL} \mathrm{~min}{ }^{-1}$ and the sparge rate for the helium flow $100 \mathrm{~mL} \mathrm{~min}{ }^{-1}$. Two eluents were used: $1 \%$ formic acid (A) and methanol (B). The gradient was the following: $0-5$ min, 100\% A; 5-180 min, $0-40 \%$ B in A; $180-220 \mathrm{~min}, 40-60 \% \mathrm{~B}$ in $\mathrm{A} ; 220-240 \mathrm{~min}, 60-80 \% \mathrm{~B}$ in $\mathrm{A}$. The injection volume was $5 \mathrm{~mL}$. The photodiode array detector was operating between 190-500 nm, and ellagitannins were detected at $280 \mathrm{~nm}$. The final purification of ellagitannins was performed by semipreparative HPLC with the same HPLC-DAD system. The column was a Gemini C18 column $(150 \times 21.2 \mathrm{~mm}, 10 \mu \mathrm{m}$, Phenomenex $)$ and the eluents were $0.1 \%$ formic acid (A) and acetonitrile (B). Different gradients were used for different oligomers; for example, a typical gradient for larger oligomers was as follows: $0-5 \mathrm{~min}, 17 \% \mathrm{~B}$ in $\mathrm{A} ; 5-51 \mathrm{~min}, 17-47 \% \mathrm{~B}$ in $\mathrm{A} ; 51-55 \mathrm{~min}, 47-70 \% \mathrm{~B}$ in A. The flow rate was $8 \mathrm{~mL} \mathrm{~min}{ }^{-1}$ and the sparge rate for the helium flow $100 \mathrm{~mL} \mathrm{~min}{ }^{-1}$. The injection volume was $5 \mathrm{~mL}$. The photodiode array detector was operating between $190-500 \mathrm{~nm}$, and ellagitannins were detected at $280 \mathrm{~nm}$.

All steps in the extraction, isolation and preparative and semipreparative purifications were followed by UPLC-DAD-MS (Acquity UPLC®, Waters Corporation, Milford, USA 
117 combined with Xevo® TQ, Waters Corporation, Milford, USA). Samples were filtered with a 118 syringe filter ( $4 \mathrm{~mm}, 0.2 \mu \mathrm{m}$ PTFE, Thermo Fisher Scientific Inc., Waltham, USA) prior to the 119 analysis. The Acquity UPLC ${ }^{\circ}$ BEH Phenyl column $(2.1 \times 100 \mathrm{~mm}, 1.7 \mu \mathrm{m}$, Waters Corporation, 120 Wexford, Ireland) was used with two eluents: $0.1 \%$ formic acid (A) and acetonitrile (B). The 121 gradient was 0-0.5 min, 0.1\% B in A; 0.5-5.0 min, 0.1-30.0\% B in A (linear gradient); 5.0-5.1 122 min, 30.0-90.0 \% B in A (linear gradient); 5.1-8.5 min, column wash and stabilization. The flow rate was $0.5 \mathrm{~mL} \mathrm{~min}{ }^{-1}$ and the injection volume $5 \mu \mathrm{L}$. The photodiode array detector was operating between 190-500 nm, and ellagitannins were detected at $280 \mathrm{~nm}$. Mass spectrometer was operated in a negative ionization mode and ions at $m / z \quad 160-1200$ were scanned. Capillary voltage was set at $3.53 \mathrm{kV}$, cone voltage ramp was used between $20-70 \mathrm{~V}$, desolvation temperature was set at $650{ }^{\circ} \mathrm{C}$, and source temperature at $150{ }^{\circ} \mathrm{C}$. Desolvation and cone gas $\left(\mathrm{N}_{2}\right)$ flow rates were $1000 \mathrm{~L} \mathrm{~h}^{-1}$ and $100 \mathrm{~L} \mathrm{~h}^{-}$, respectively. The ellagitannins were identified based on previous work. ${ }^{3,36}$ Pure ellagitannins were concentrated to the water-phase and freeze-dried. 98\%, lyophilized powder, $66 \mathrm{kDa}$; Sigma-Aldrich, St. Louis, US) were performed with a NanoITC 132 instrument (TA Instruments Ltd., Crawley, West Sussex, UK) as previously described ${ }^{18,23,24}$ with minor modifications. All solutions were prepared in $50 \mathrm{mM}$ citrate buffer adjusted to $\mathrm{pH} 6$. BSA solution $(10,20,30$ or $40 \mu \mathrm{M})$ was placed in the $950 \mu \mathrm{L}$ sample cell of the calorimeter and $3 \mathrm{mM}$ 135 ellagitannin solution was loaded into the injection syringe. The ellagitannin studied was titrated into 136 the sample cell at $298 \mathrm{~K}$ as a sequence of 24 injections of $10 \mu \mathrm{L}$. The time delay between the 137 injections was $360 \mathrm{~s}$. The content of the sample cell was stirred throughout the experiment to ensure 138 comprehensive mixing. All ellagitannins were studied with three replicate titrations; the pentameric 139 and heptameric ellagitannins were studied in duplicates because of limited amounts. Control 140 experiments included the titration of ellagitannin solution into buffer, the titration of buffer into 141 BSA solution and the titration of buffer into buffer. Control experiments of buffer titrated into BSA 
142

143

144

146

151

152

153

154

155

156

157

158

159

160

161

162

163

164

165

166

solution and buffer into buffer resulted only in small or equal enthalpy changes for each successive injection of buffer, and therefore, were not considered in the data analysis. ${ }^{18,23,24}$ The control data of ellagitannin titrated into buffer was always subtracted from the sample data as it was known that ellagitannins tend to self-associate into aggregates due to hydrophobic groups; and therefore, when injected from the syringe into buffer, they undergo an endothermic process of deaggregation. The extent of deaggregation depends inversely on the concentration of ellagitannin already present in the sample cell: therefore, successive injections of ellagitannins into buffer lead to observation of progressively lower endothermic enthalpy changes as has been illustrated in earlier work. ${ }^{22}$

Data Analysis. Raw data from isothermal titration calorimetry were obtained as plots of heat $(\mu \mathrm{J})$ against injection number and exhibited a series of peaks for each injection. The raw data were transformed using the NanoAnalyze Data Analysis software (version 2.4.1., TA Instruments) to obtain a plot of observed enthalpy change per mole of injectant $\left(\Delta \mathrm{H}_{\mathrm{obs}}, \mathrm{kJ} \mathrm{mol}^{-1}\right)$ against molar ellagitannin:protein ratio. The estimated binding parameters were obtained from ITC data using the same NanoAnalyze Data Analysis software. Data fits were obtained in two different ways: using a single set of multiple binding sites and a model for two independent sets of multiple binding sites. The quality of fits was determined by standard deviation.

\section{RESULTS AND DISCUSSION}

ITC Binding Isotherms and Data Fitting. In this study, the interaction of seven individual tellimagrandin I-based oligomeric ellagitannins (from monomer to heptamer) and a mixture of larger oligomeric ellagitannins with BSA was investigated by ITC. For each ellagitannin:BSA system studied, an exothermic interaction was observed. Fig. 2 shows ITC binding isotherms for two of the ellagitannins studied, the trimer (oenothein A) and hexamer binding to BSA. Experiments were carried out using two different concentrations of BSA, $20 \mu \mathrm{M}$ or 
167

168

169

170

171

172

173

176

177

178

179

180

181

182

183

184

185

186

187

188

189

190

191

$30 \mu \mathrm{M}$, and this showed the good repeatability of the ellagitannin:BSA interaction. The data showed that the interaction was not affected by BSA concentration suggesting no evidence of cooperative binding. Upon the addition of ellagitannin, the interaction became less exothermic as the binding sites of BSA became saturated. The ITC binding isotherms showed that an ellagitannin:BSA molar ratio of approximately 10:1 corresponded to the inflection point for each ellagitannin interaction and that there were multiple binding sites for the ellagitannin on the BSA molecule. $^{18}$

Two different binding models were used to fit the data, one assuming a single set of multiple binding sites (a single-site model) and a second model assuming two independent sets of multiple binding sites (a two-site model), as previously discussed in detail by Deaville et al. ${ }^{25}$ In Fig. 3, it can be seen that both models fit the data equally well for the ellagitannin trimer binding to BSA, and thus the simpler single-site model seemed better justified as a binding model. However, for many of the ellagitannins studied, both models provided an acceptable fit, but the two-site model clearly exhibited a closer fit for the data. This was seen particularly at higher ellagitannin:BSA molar ratios where a longer tail in the data was seen as the tannin:BSA interaction was saturated. Therefore, the fit parameters for both binding models are shown in Tables 1 and 2 for all ellagitannins studied. The second site showed very weak interaction with small binding constants varying from 17 to $1.8 \times 10^{3} \mathrm{M}^{-1}$ (Table 2) for all the ellagitannin:BSA interactions studied. This weaker interaction might indicate a non-specific surface adsorption mechanism following the more selective stronger binding of the ellagitannin, as indeed we had observed in previous tannin:protein binding studies. ${ }^{22-24}$ This was the first study where a purified oligomeric series was investigated to look at the effect of oligomerization on ellagitannin:protein binding behavior. In previous studies with hydrolysable tannins, a two-binding site model had been used. In those examples, the binding constants had shown to be higher than values seen here, and the twosite model had been more pronounced. Interestingly, those measurements had been carried out 
192 using lower tannin concentrations $\left(3 \mathrm{mg} \mathrm{mL}^{-1}\right.$, for example approximately $2 \mathrm{mM}$ for oenothein $\left.\mathrm{B}\right)$

193 and over a longer timescale using more injections and a higher level of protein dilution than the

194 current experiments. From our experience it was clear that we could not directly compare data from

195 previous studies, which has used different experimental conditions that could impact on the

196 interaction, due to complicating factors such as the degree of self-association of tannin molecules.

The two-site model indicated two independent types of binding on the protein surface.

198 The second binding site highlighted the presence of a weaker interaction in addition to a more 199 selective stronger first binding site. As seen in Table 2, the weakness of the second binding site 200 meant that it was difficult to confidently identify trends across the oligomeric series for this 201 interaction. Therefore, we have focused on the single-binding site model for our discussion of the 202 ellagitannin:BSA interaction across the oligomeric series since this interaction was likely to relate 203 to a specific molecular interaction between the tannin and protein. linking the oligomer size to ellagitannin:BSA interaction, that suggested that the smaller ellagitannins deviated from the overall trends seen. The interaction of monomeric tellimagrandin I with BSA was stronger, in terms of $K_{a}$ than expected in relation to the oligomer series. The binding constant $\left(K_{a}=1.8 \times 10^{4} \mathrm{M}^{-1}\right)$ was higher than for the dimeric oenothein $\mathrm{B}\left(5.7 \times 10^{3} \mathrm{M}^{-1}\right)$ and trimer $\left(7.6 \times 10^{3} \mathrm{M}^{-1}\right)$ and similar to hexameric and heptameric ellagitannins $\left(1.7 \times 10^{4} \mathrm{M}^{-1}\right)$. This could be due to the additional free galloyl group as the monomer contained two adjacent free galloyl groups (Fig. 1A) whereas oligomeric ellagitannins contained only one free galloyl group per monomeric unit. Previously, Dobreva et al. had reported that the strong binding site was dependent on tannin flexibility and possibly also free galloyl groups. ${ }^{24}$ 
217 Oenothein B was a macrocyclic, relatively rigid structure with less conformational flexibility 218 compared with all the other ellagitannins studied in this series (Fig. 1B). A previous study had 219 shown that the binding constant for the interaction with BSA was dependent on the structural 220 flexibility of the tannin molecule; and a loss of conformational freedom in the ellagitannin structure 221 impacted on its ability to bind to BSA. ${ }^{25}$ The thermodynamic parameters for oenothein B (Table 1 222 and 2) were different to Dobreva et al. ${ }^{24}$ although the overall conclusion linking BSA binding to 223 tannin flexibility was the same. As mentioned previously, differences in the experimental 224 procedures were likely to explain the quantitative difference in the binding interaction. ${ }^{24}$ Our 225 findings suggested that the interaction between ellagitannin and protein might be dependent on the 226 ellagitannin concentration.

The trimeric oligomer, oenothein A, showed stronger binding to BSA than the dimer.

228 The binding constant was $7.5 \times 10^{3} \mathrm{M}^{-1}$ and the enthalpy change was $-45 \mathrm{~kJ} \mathrm{~mol}^{-1}$. The trimer was 229 more flexible than the dimer as the additional monomeric unit was attached only via one bond (Fig. 1C). The presence of this conformationally free chain (flexible tail) allowed for a stronger interaction in terms of $\mathrm{K}_{\mathrm{a}}$ of the trimer versus the dimer and this was also seen for the larger 232 oligomers in terms of $\Delta \mathrm{H}_{\mathrm{obs}}$ (Fig. 4). These observations could be linked to the three-dimensional 233 structures of the oligomer series, where larger oligomers had a longer flexible chain attached to the 234 macrocycle.

Larger Oligomeric Ellagitannins. The interactions of ellagitannin oligomers from trimers to octamer-undecamers with BSA revealed strong similarities. The stoichiometric number,

237 i.e. tannin to protein ratio, varied from just 9 to 12 (Table 1) and the equilibrium binding constant 238 was $10^{3}-10^{4} \mathrm{M}^{-1}$ for all ellagitannin-BSA interactions. The observed change in enthalpy was 239 exothermic and increased with the oligomeric size up to the heptamer. Exothermic interactions were 240 associated with hydrogen bonding or Van der Waals interactions. As the oligomeric size increased 241 the number of footholds, the points of interaction with the protein surface, increased. 
All oligomers contained the same macrocyclic part in their structure, i.e. dimeric oenothein B (Fig. 1B) formed by the dimerization of two tellimagrandin I monomers via two $\mathrm{m}$ -

244 DOG-type linkages. The $m$-DOG-type linkages are frequently found in ellagitannin oligomers: in these linking units, the $O$-donating hydroxyl group is part of an hexahydroxydiphenoyl group and the galloyl group is the acceptor. ${ }^{2}$ In trimeric and larger ellagitannin oligomers, the additional monomeric units were attached by one $m$-DOG-type linkage forming an elongated chain or tail to this macrocyclic part (Fig. 1). It was likely that first the macrocyclic part of the ellagitannin bound to the protein (to the defined binding sites on the protein surface resulting in little difference in the value of $\mathrm{n}$ for each tannin) and then the "flexible tail" of the ellagitannin coated the protein surface. Therefore, a trend in terms of binding affinity could be observed for this oligomeric ET series. This observation differed from the previous studies of polyphenol binding to proteins where an increase in the binding affinity with molecular size had been observed, but where there were also differences in terms of flexibility, molecular structures and in some cases, the purity of the tannins that 255 influenced the interaction. ${ }^{38}$ effect of oligomerization and molecular weight, the data were also fitted by assuming a monomeric concentration for the ellagitannins as shown in Table 3 using a single-site binding model. The oligomers consisted of similar monomeric units, which enabled the direct comparison of the different oligomers based on the number of monomer units. By doing this, the trend seen in Table 1

261 for $\Delta \mathrm{H}_{\mathrm{obs}}$ was largely removed. Focusing on the dimer and larger oligomers the variation seen for $262 \Delta \mathrm{H}$ was small at $-13.9 \pm 2.6 \mathrm{~kJ}$ per mole of monomer. However, the number of binding sites on the 263 ellagitannin increased with the oligomer size, since the binding of the flexible chain was now 264 considered as individual molecules. We saw that the $K_{a}$ was smaller per monomer compared with 265 our molecular calculations, since the interaction was now split across multiple molecules. 
The different analyses of the data seemed to fit the concept that there were two

267 different binding events; first the binding of the rigid ring of the dimer was followed by the binding

268 of the flexible units of the tail. It was for this reason why in previous studies two-site binding models had been needed and why we fitted our current data also using the two-site binding model (Table 2). However, often the second binding site was a lot weaker and less specific than the first one. As mentioned previously, the fits obtained using a two-site model were good fits, and marginally better than the single-site model for the larger ellagitannins. In particular, oligomers from tetramer to heptamer all showed good two-site binding fits to the data with nine distinct strong binding sites $\left(n=9, K_{a}=10^{4}-10^{5} \mathrm{M}^{-1}\right)$ and a second weak binding site, a $K_{a}$ of approximately $10^{2}$ and a high $n$ ranging from 35-90.

Previous studies had shown that the interaction of tannins with proteins could be a surface phenomenon where tannins coated the surface of the protein. ${ }^{18,25,39}$ Our data supported this observation in two ways. Firstly, the flexible elongated chain in oligomeric ellagitannins appeared to coat the protein surface. Secondly, when the data were fitted using monomeric concentrations, the tannin:protein binding stoichiometries increased $\mathrm{x}$-fold, where $\mathrm{x}$ was the number for the degree of oligomerization, and the $\Delta \mathrm{H}$ and $K_{a}$ values converged. We observed that there were approximately nine specific binding sites on the surface of the protein, but that further interactions, akin to non-specific surface adsorption, occurred allowing the flexible chain of the tannin oligomers to subsequently bind to the surface.

In conclusion, this unique series of oligomeric ellagitannins allowed us to study the effect of molecular size on the interaction between ellagitannins and BSA. The novel results showed that the interactions of ellagitannin oligomers from trimers to octamer-undecamers with BSA revealed strong similarities. The monomeric and dimeric ellagitannins deviated from the overall trends seen. Our studies highlighted the importance of molecular flexibility to maximize binding between the tannin and protein surface. This systematic investigation of ellagitannins used 
an oligomeric series and was able to decouple for the first time structural features, such as

292 functional groups present and purity, from molecular weight.

293

294

\section{ABBREVIATIONS USED}

295

BSA, bovine serum albumin; HPLC-DAD, high-performance liquid chromatography diode array detection; ITC, isothermal titration calorimetry; UPLC-DAD-MS, ultra-performance liquid chromatography diode array detection mass spectrometry

\section{ACKNOWLEDGEMENTS}

We thank Mika Karonen for his help in the collection of plant materials and Atte Tuominen and

Hanna-Mari Salmia for their help in the isolation and purification of ellagitannins. Marina Dobreva is acknowledged for her help with the ITC measurements.

\section{REFERENCES}

305

(1) Okuda, T.; Yoshida, T.; Hatano, T.; Ito, H. Ellagitannins renewed the concept of tannins. In Chemistry and Biology Ellagitannins: An underestimated class of bioactive plant polyphenols; Quideau, S, Ed.; World Scientific Publishing, Singapore, 2009; pp. 1-54.

(2) Yoshida, T.; Hatano, T.; Ito, H.; Okuda, T. Structural diversity and antimicrobial activities of ellagitannins. In Chemistry and Biology Ellagitannins: An underestimated class of bioactive plant polyphenols; Quideau, S, Ed.; World Scientific Publishing, Singapore, 2009; pp. 55-93.

(3) Karonen, M.; Parker, J.; Agrawal, A.; Salminen, J.-P. First evidence of hexameric and heptameric ellagitannins in plants detected by liquid chromatography/electrospray ionisation mass spectrometry. Rapid Commun. Mass Spectrom. 2010, 24, 3151-3156. 
(4) Salminen, J.-P.; Karonen, M. Chemical ecology of tannins and other phenolics: we need a change in approach. Funct. Ecol. 2011, 25, 325-338.

(5) Quideau, S.; Deffieux, D.; Douat-Casassus, C.; Pouyséqu, L. Plant polyphenols: chemical properties, biological activities, and synthesis. Angew. Chem. Int. Ed. 2011, 50, 586-621.

(6) Lowry, J.B.; McSweeney, C.S.; Palmer, B. Changing perceptions of the effect of plant phenolics on nutrient supply in the ruminant. Aust. J. Agric. Res. 1996, 47, 829-842.

(7) McNeill, D.M.; Osborne, N.J.; Komolong, M.; Nankervis, D. Condensed tannins in the genus Leucaena and their nutritional significance for ruminants. ACIAR Proc. 1998, 86, $205-214$

(8) Min, B.R.; Barry, T.N.; Attwood, G.T.; McNabb, W.C. The effect of condensed tannins on the nutrition and health of ruminants fed fresh temperate forages: a review. Anim. Feed Sci. Technol. 2003, 106, 3-19.

(9) Waghorn, G.C.; McNabb, W.C. Consequences of plant phenolic compounds for productivity and health of ruminants. Proc. Nutr. Soc. 2003, 62, 383-392.

(10) Kebreab, E.; Mills, J.A.N.; Crompton, L.A.; Bannik, A.; Dijkstra, J.; Gerrits, W.J.J. An integrated mathematical model to evaluate nutrient partition in dairy cattle between animal and environment. Anim. Feed Sci. Technol. 2004, 112, 131-154.

(11) Givens, D.I.; Rulquin, H. Utilisation by ruminants of nitrogen compounds in silage-

(13) McWilliam, E.L.; Barry, T.N.; López-Villalobos, N.; Cameron, P.N.; Kemp, P.D. 
of ewes grazing low quality drought pasture during mating. Anim. Feed Sci. Technol. 2005, 119, 87-106.

(14) Mlambo, V.; Smith, T.; Owen, E.; Mould, F.L; Sikosana, J.L.N.; Mueller-Harvey, I. Tanniniferous Dichrostachys cinerea fruits do not require detoxification for goat nutrition: in sacco and in vivo evaluations. Livestock Prod. Sci. 2004, 90, 135-144.

(15) Mueller-Harvey, I. Unravelling the conundrum of tannins in animal nutrition and health. J. Agric. Food Chem. 2006, 86, 2010-2037.

(16) Mueller-Harvey, I.; Mlambo, V.; Sikosana, J.L.N.; Smith, T.; Owen, E.; Brown, R.H. Octanol-water partition coefficients for predicting the effects of tannins in ruminant nutrition. J. Agric. Food Chem. 2007, 55, 5436-5444.

(17) Asquith, T.N.; Butler, L.G. Interactions of condensed tannins with selected proteins. Phytochemistry 1986, 25, 1591-1593.

(18) Dobreva, M.A.; Frazier, R.A.; Mueller-Harvey, I.; Clifton, L.A.; Gea, A.; Green, R.J.

Binding of pentagalloyl glucose to two globular proteins occurs via multiple surface sites. Biomacromolecules 2011, 12, 710-715.

(19) Hagerman, A.E.; Butler, L.G. The specificity of proanthocyanidin-protein interactions. J. Biol. Chem. 1981, 4494-4497.

(20) Lewis, E.A.; Murphy, K.P. Isothermal titration calorimetry. In Methods in Molecular Biology: Protein-ligand interactions: Methods and applications, Nienhaus, G.U., Ed.; Humana Press Inc., Totowa, 2005; 305, pp. 1-15.

(21) Ghai, R.; Falconer, R.J.; Collins, B.M. Applications of isothermal titration calorimetry in pure and applied research-survey of the literature from 2010. J. Mol. Recognit. 2012, 25, $32-52$. 
(22) Frazier, R.A.; Papadopoulou, A.; Mueller-Harvey, I.; Kissoon, D.; Green, R.J. Probing protein-tannin interactions by isothermal titration microcalorimetry. J. Agric. Food Chem. 2003, 51, 5189-5195.

(23) Frazier, R.A.; Deaville, E.R.; Green, R.J.; Stringano, E.; Willoughby, I.; Plant, J.;

Mueller-Harvey, I. Interaction of tea tannins and condensed tannins with proteins. J. Pharm. Biomed. Anal. 2010, 51, 490-495.

(24) Dobreva, M.A.; Green, R.J.; Mueller-Harvey, I.; Salminen, J.-P.; Howlin, B.J.;

Frazier, R.A. Size and molecular weight affect the binding of ellagitannins to bovine serum albumin. J. Agric. Food Chem. 2014, 62, 9186-9194.

(25) Deaville, E.R.; Green, R.J.; Mueller-Harvey, I.; Willoughby, I.; Frazier, R.A. Hydrolyzable tannin structures influence relative globular and random coil protein binding strengths. J. Agric. Food Chem. 2007, 55, 4554-4561.

(26) Kilmister, R.L.; Faulkner, P.; Downey, M.O.; Darby, S.J.; Falconer, R.J. The

(27) Guyot, S.; Doco, T.; Souquet, J.-M.; Moutounet, M.; Drilleau, J.-F. Characterization of highly polymerized procyanidins in cider apple (Malus sylvestris var. Kermerrien) skin and pulp. Phytochemistry 1997, 44, 351-357.

(28) Guyot, S.; Matner, N.; Laraba, D.; Sanoner, P.; Drilleau, J.-F. Reversed-phase HPLC

(29) Karonen, M.; Loponen, J.; Ossipov, V.; Pihlaja, K. Analysis of procyanidins in pine bark with reversed-phase and normal-phase high-performance liquid chromatographyelectrospray ionization mass spectrometry. Anal. Chim. Acta 2004, 522, 105-112. 
(30) Thompson, R.S.; Jacques, D.; Haslam, E.; Tanner, R.J.N. Plant proanthocyanidins. Part I. Introduction; the isolation, structure and distribution in nature of plant procyanidins. J. Chem. Soc., Perkin Trans. 1 1972, 11, 1387-1399.

(31) Guyot, S.; Marnet, N.; Sanoner, P.; Drilleau, J.-F. Direct thiolysis on crude apple materials for high-performance liquid chromatography characterization and quantification of polyphenols in cider apple tissues and juices. Methods Enzymol. 2001, 335, 57-70.

(32) Matthews, S.; Mila, I.; Scalbert, A.; Pollet, B.; Lapierre, C.; Hervé du Penhoat,

C.L.M.; Rolando, C.; Donnelly, D.M.X. Method for estimation of proanthocyanidins based on their acid depolymerization in the presence of nucleophiles. J. Agric. Food Chem. 1997, $45,1195-1201$.

(33) Ohnishi-Kameyama, M.; Yanagida, A.; Kanda, A.; Nagata, T. Identification of 400

(34) Vivas, N.; Nonier, M.-F.; Vivas de Gaulejac, N.; Absalon, C.; Bertrand, A.; Mirabel,

M. Differentiation of proanthocyanidin tannins from seeds, skins and stems of grapes (Vitis vinifera) and heartwood of Quebracho (Schinopsis balansae) by matrix-assisted laser desorption/ionization time-of-flight mass spectrometry and thioacidolysis/liquid chromatography/electrospray ionization mass spectrometry. Anal. Chim. Acta 2004, 513, $247-256$.

(35) Karonen, M.; Leikas, A.; Loponen, J.; Sinkkonen, J.; Ossipov, V.; Pihlaja, K.

Reversed-phase HPLC-ESI/MS analysis of birch leaf proanthocyanidins after their acidic degradation in the presence of nucleophiles. Phytochem. Anal. 2007, 18, 378-386. 
(36) Moilanen, J.; Salminen, J.-P. Ecologically neglected tannins and their biologically relevant activity: chemical structures of plant ellagitannins reveal their in vitro oxidative activity at high $\mathrm{pH}$. Chemoecology 2008, 18, 73-83.

(37) Baert, N.; Karonen, M.; Salminen, J.-P. 2015. Isolation, characterisation and quantification of the main oligomeric macrocyclic ellagitannins in Epilobium angustifolium by ultra-high performance chromatography with diode array detection and electrospray

(39) Spencer, C.M.; Cai, Y.; Russell, M.; Gaffney, S.H.; Goulding, P.N.: Magnolato, D.;

Lilley, T.H.; Haslam, E. Polyphenol complexation-some thoughts and observations. Phytochemistry 1988, 27, 2397-2409.

\section{Funding}

This research was funded by Academy of Finland (grant 251388 to author MK). Authors IM-H and J-PS received funding through a Marie Curie Initial Training Network from the European Union ('LegumePlus', PITN-GA-2011-289377). Analyses and characterization of ellagitannins on the University of Turku (Ecological Interactions).

\section{$431 \quad$ Notes}

432 The authors declare no competing financial interest. 
FIGURE CAPTIONS

435

436

Figure 1. The structures of (A) monomeric ellagitannin tellimagrandin I, (B) dimeric ellagitannin oenothein B, (C) trimeric ellagitannin oenothein A, and (D) tetrameric tellimagrandin I-based 438 ellagitannin.

439

Figure 2. Typical ITC binding isotherms for the interaction of ellagitannin (A) trimer (oenothein A) and (B) hexamer with $20 \mu \mathrm{M}$ BSA ( $(\mathbf{)})$ and $30 \mu \mathrm{M}$ BSA in two replicate experiments $(\Delta$ and $\times)$.

442

Figure 3. Single-site (short dashed line) and two-site (long dashed line) binding models fitted to the experimental data ( $\mathbf{a})$ for the interaction of ellagitannin (A) monomer (tellimagrandin I), (B) dimer 445 (oenothein B), (C) trimer, (D) tetramer, (E) pentamer, (F) hexamer, $(\mathrm{G})$ heptamer and $(\mathrm{H})$ a mixture 446 of octamer-undecamers with $30 \mu \mathrm{M}$ BSA.

Figure 4. Plots of (A) $\Delta \mathrm{H}$ and (B) $\mathrm{K}_{\mathrm{a}}$ vs degree of oligomerization for the interaction of 449 ellagitannin oligomers with BSA fitted by single-site binding model. 
Table 1. Estimated Thermodynamic Binding Parameters for the Interaction of Ellagitannin Oligomers with BSA Fitted by a Single-site Binding Model ${ }^{a}$

\begin{tabular}{|c|c|c|c|c|c|c|c|c|}
\hline & Monomer & Dimer & Trimer & Tetramer & Pentamer & Hexamer & Heptamer & $\begin{array}{l}\text { Octamer- } \\
\text { undecamers }\end{array}$ \\
\hline$\overline{\mathrm{K}_{\mathrm{a} 1}^{b}}$ & $18403 \pm 5052$ & $5739 \pm 794$ & $7552 \pm 136$ & $26810 \pm 2708$ & 23200 & $16925 \pm 551$ & 16925 & 35520 \\
\hline$\Delta \mathrm{H}_{1}{ }^{c}$ & $-24 \pm 3$ & $-14 \pm 4$ & $-45 \pm 5$ & $-69 \pm 3$ & -80 & $-88 \pm 3$ & -93 & -93 \\
\hline $\mathrm{n}_{1}$ & $6 \pm 1$ & $12 \pm 2$ & $11 \pm 1$ & $9 \pm 1$ & 9 & $10 \pm 1$ & 10 & 9 \\
\hline SD & 13 & 11 & 11 & 71 & & 94 & & \\
\hline
\end{tabular}

${ }^{a} \mathrm{SD}=$ standard deviation around fit obtained by NanoAnalyze software; $\mathrm{n}=3$, except for ellagitannin pentamer, heptamer and octamer to undecamer $\mathrm{n}=2$ due to the limited amount of individual oligomers. ${ }^{b} \mathrm{~K}_{\mathrm{a} 1}\left(\mathrm{M}^{-1}\right)$ is the equilibrium binding constant for the single set of multiple binding sites. ${ }^{c} \Delta \mathrm{H}_{1}\left(\mathrm{~kJ} \mathrm{~mol}^{-1}\right)$ is the corresponding enthalpy. 
Table 2. Estimated Thermodynamic Binding Parameters for the Interaction of Ellagitannin Oligomers with BSA Fitted by a Two-site Binding Model ${ }^{a}$

\begin{tabular}{|c|c|c|c|c|c|c|c|c|}
\hline & Monomer & Dimer & Trimer & Tetramer & Pentamer & Hexamer & Heptamer & $\begin{array}{l}\text { Octamer- } \\
\text { undecamer }\end{array}$ \\
\hline $\mathrm{K}_{\mathrm{a} 1}^{b}$ & $22188 \pm 6280$ & $9786 \pm 1597$ & $11863 \pm 1090$ & $\begin{array}{r}72560 \pm \\
10124\end{array}$ & 76620 & $55890 \pm 5419$ & 35280 & 42940 \\
\hline$\Delta \mathrm{H}_{1}^{c}$ & $-20 \pm 3$ & $-14 \pm 1$ & $-33 \pm 3$ & $-52 \pm 4$ & -56 & $-63 \pm 5$ & -73 & -88 \\
\hline $\mathrm{n}_{1}$ & $6 \pm 2$ & $6 \pm 1$ & $11 \pm 1$ & $9 \pm 1$ & 9 & $9 \pm 1$ & 9 & 9 \\
\hline $\mathrm{K}_{\mathrm{a} 2}{ }^{b}$ & $1828 \pm 1815$ & $1138 \pm 998$ & $350 \pm 93$ & $184 \pm 19$ & 262 & $115 \pm 11$ & 174 & 17 \\
\hline$\Delta \mathrm{H}_{2}{ }^{c}$ & $-10 \pm 9$ & $-7 \pm 3$ & $-9 \pm 3$ & $-19 \pm 3$ & -44 & $-59 \pm 2$ & -27 & -24 \\
\hline $\mathrm{n}_{2}$ & $4 \pm 1$ & $36 \pm 12$ & $32 \pm 14$ & $90 \pm 11$ & 35 & $59 \pm 1$ & 84 & 83 \\
\hline SD & 12 & 7 & 14 & 50 & & 73 & & \\
\hline
\end{tabular}

${ }^{a} \mathrm{SD}=$ standard deviation around fit obtained by NanoAnalyze software; $\mathrm{n}=3$, except for ellagitannin pentamer, heptamer and octamerundecamer $\mathrm{n}=2$ due to the limited amount of individual oligomers. ${ }^{b} \mathrm{~K}_{\mathrm{a} 1}$ and $\mathrm{K}_{\mathrm{a} 2}\left(\mathrm{M}^{-1}\right)$ are the equilibrium binding constants for the two set of multiple binding sites. ${ }^{c} \Delta \mathrm{H}_{1}$ and $\Delta \mathrm{H}_{2}\left(\mathrm{~kJ} \mathrm{~mol}^{-1}\right)$ are the corresponding enthalpies. 
Table 3. Estimated Thermodynamic Binding Parameters for the Interaction of Ellagitannin Oligomers with BSA Fitted by a Single-site Binding Model. The Concentrations of Oligomers Have Been Set to the Corresponding Monomeric Concentrations in Order to Remove the Impact of Molecular Weight ${ }^{a}$

\begin{tabular}{|c|c|c|c|c|c|c|c|c|}
\hline & Monomer & Dimer & Trimer & Tetramer & Pentamer & Hexamer & Heptamer & $\begin{array}{l}\text { Octamer- } \\
\text { undecamer }\end{array}$ \\
\hline $\mathrm{K}_{\mathrm{a} 1}^{b}$ & $18403 \pm 8750$ & $2391 \pm 1364$ & $2517 \pm 79$ & $6703 \pm 1182$ & $4645 \pm 368$ & $3082 \pm 372$ & $2420 \pm 135$ & $5119 \pm 2760$ \\
\hline$\Delta \mathrm{H}_{1}{ }^{c}$ & $-24 \pm 6$ & $-12 \pm 11$ & $-15 \pm 3$ & $-17 \pm 1$ & $-16 \pm 1$ & $-15 \pm 1$ & $-13 \pm 1$ & $-9 \pm 1$ \\
\hline $\mathrm{n}_{1}$ & $6 \pm 1$ & $21 \pm 13$ & $30 \pm 7$ & $37 \pm 4$ & 46 & $57 \pm 3$ & 67 & $85 \pm 8$ \\
\hline SD & 13 & 9 & 11 & 71 & & 94 & & 39 \\
\hline
\end{tabular}

${ }^{a} \mathrm{SD}=$ standard deviation around fit obtained by NanoAnalyze software; $\mathrm{n}=3$, except for ellagitannin pentamer, heptamer and octamer to undecamer $\mathrm{n}=2$ due to the limited amount of individual oligomers. ${ }^{b} \mathrm{~K}_{\mathrm{a} 1}\left(\mathrm{M}^{-1}\right)$ is the equilibrium binding constant for the single set of multiple binding sites. ${ }^{c} \Delta \mathrm{H}_{1}\left(\mathrm{~kJ} \mathrm{~mol}^{-1}\right)$ is the corresponding enthalpy. 
Figure 1.

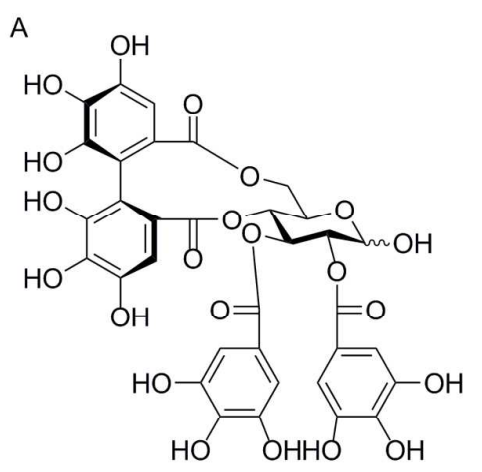

C

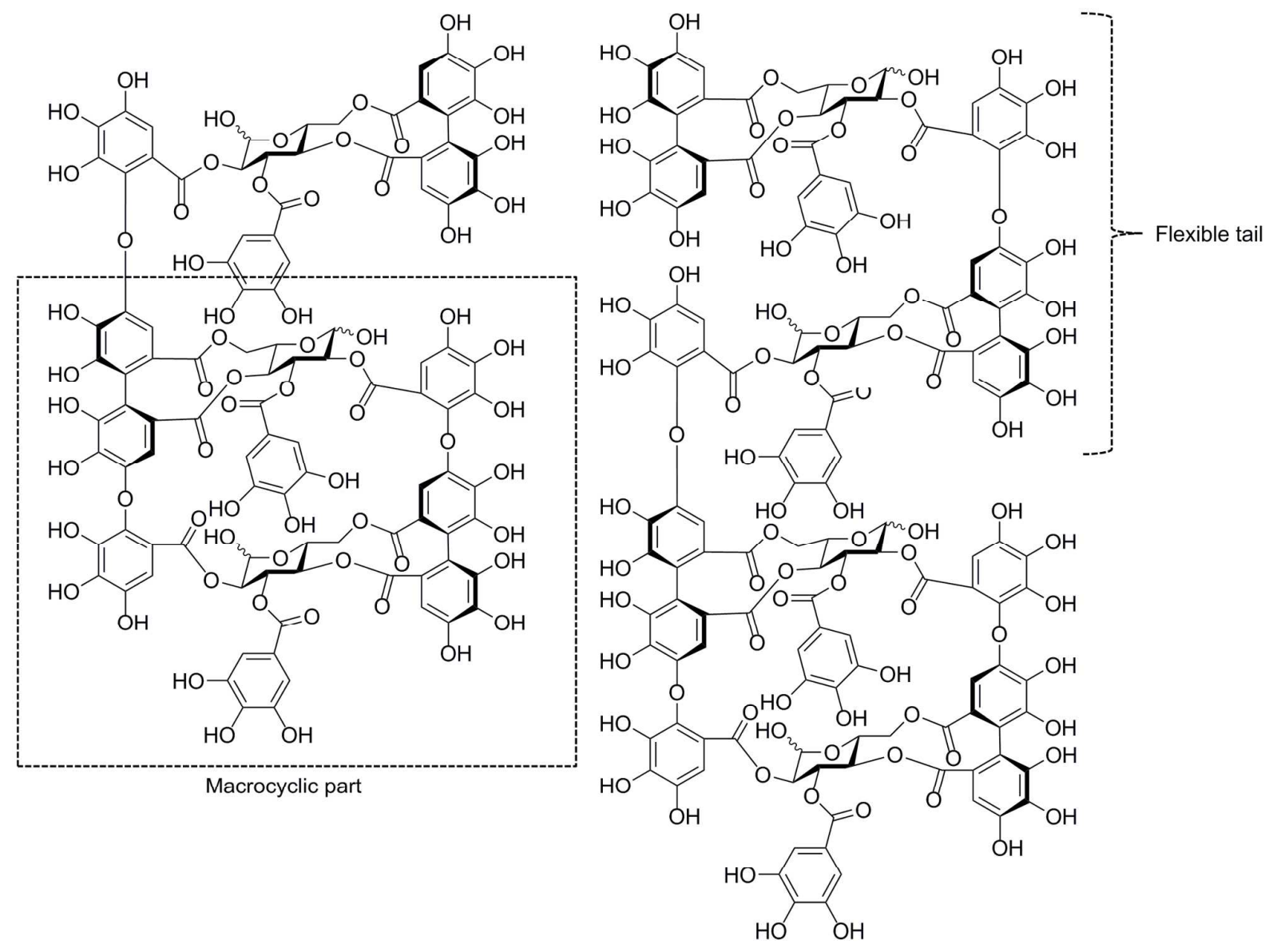


Figure 2.
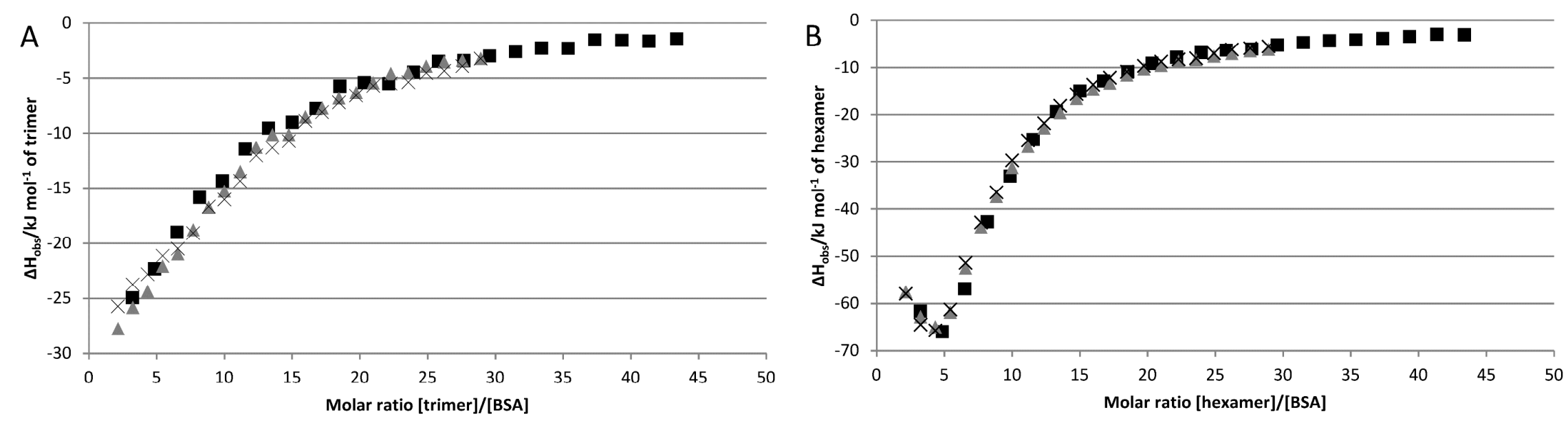
Figure 3.
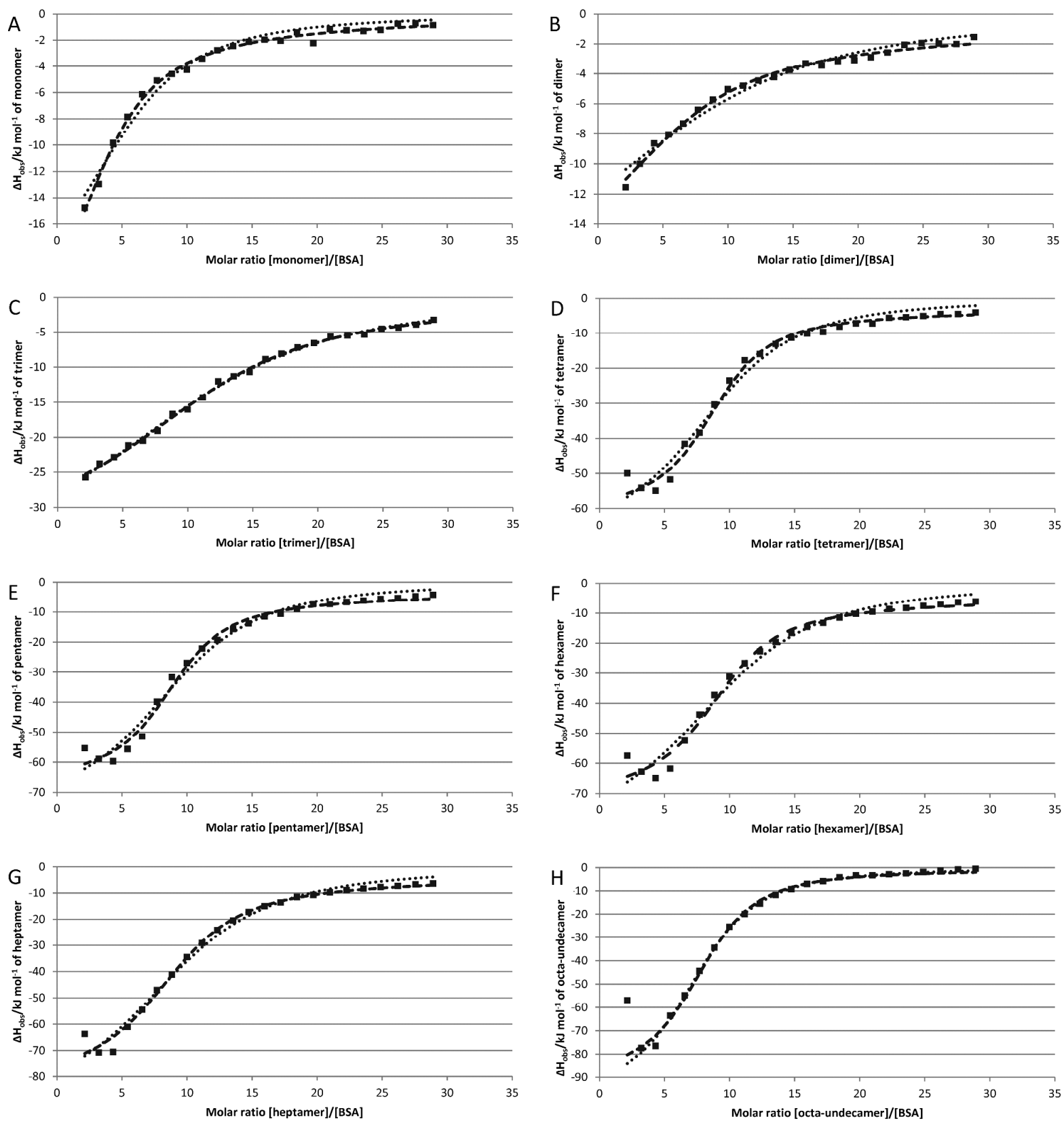
Figure 4.
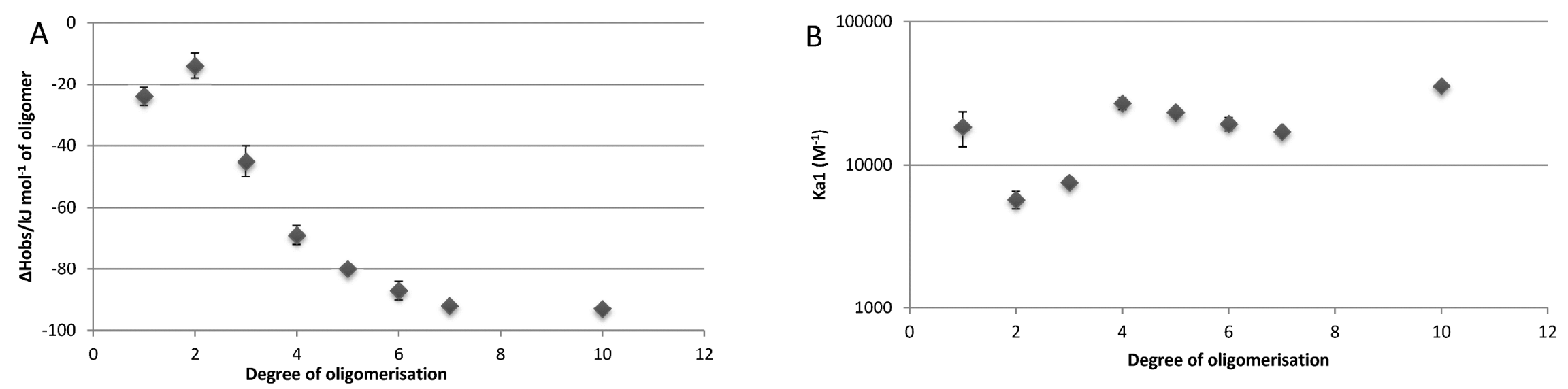
Graphic for table of contents

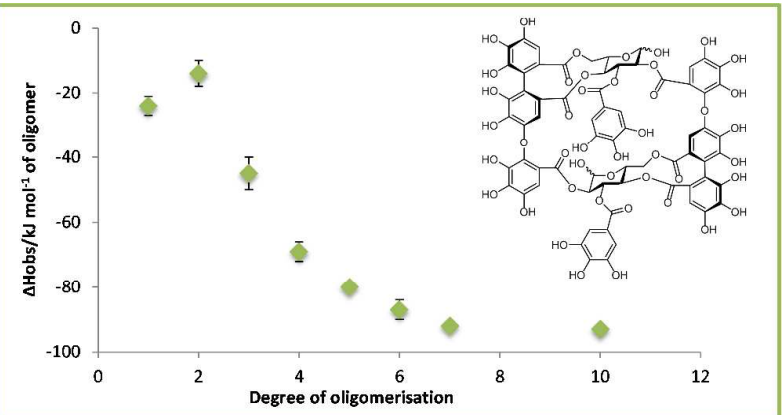

